Research Article

\title{
Translation and Validation of the Korean Version of the Postoperative Quality of Recovery Score QoR-15
}

\author{
Donggyeong Kim, ${ }^{1}$ Jay Kyoung Kim, ${ }^{2}$ and Jinseok Yeo $\mathbb{D}^{2}$ \\ ${ }^{1}$ Department of Anesthesiology and Pain Medicine, The Armed Forces Daegu Hospital, Gyeongsangbuk-do, Republic of Korea \\ ${ }^{2}$ Department of Anesthesiology and Pain Medicine, Kyungpook National University Chilgok Hospital, School of Medicine, \\ Kyungpook National University, Daegu, Republic of Korea
}

Correspondence should be addressed to Jinseok Yeo; jsyeo@knu.ac.kr

Received 7 January 2020; Revised 28 September 2020; Accepted 6 October 2020; Published 14 October 2020

Academic Editor: H.-X. Wang

Copyright (c) 2020 Donggyeong Kim et al. This is an open access article distributed under the Creative Commons Attribution License, which permits unrestricted use, distribution, and reproduction in any medium, provided the original work is properly cited.

\begin{abstract}
Quality of recovery after anesthesia is an important measure of the early postoperative health status of patients. The Quality of Recovery-15 (QoR-15) questionnaire is a self-rated questionnaire used to assess the quality of postoperative recovery. This study is aimed at translating and validating the Korean version of QoR-15 (QoR-15K). One hundred fifty patients were included in this study. We translated the original version of QoR-15 into QoR-15K and evaluated its validity, reliability, responsiveness, and clinical feasibility. QoR-15K showed acceptable criterion, structure, and construct validities. Reliability was verified using Cronbach's $\alpha$ (0.856), standard error of measurement (10.78), split-half reliability (0.831), test-retest reliability $(\rho=0.945, P \leq 0.001)$, and intraclass correlation test-retest $(\rho=0.903, P \leq 0.001)$. These results represent an acceptable reliability. Responsiveness was verified using Cohen's effect size (1.39), standardized response mean (1.03), and the correlation between QoR-15K score and duration of anesthesia $(\rho=-0.197, P=0.016)$. These results show acceptable responsiveness. The mean \pm standard deviation time to complete QoR-15K was $138.1 \pm 30.7 \mathrm{~s}$. QoR-15K was rated more than adequate on the COnsensus-based Standards for the selection of health Measurement INstruments checklist. In conclusion, QoR-15K shows acceptable validity, reliability, responsiveness, and clinical feasibility and may help evaluate postoperative quality of recovery in Korean populations.
\end{abstract}

\section{Introduction}

Recovery after surgery is a complex process. Many factors affect the recovery process, including the patient's medical condition, type of surgery, adverse sequelae after surgery, and anesthesia. With the introduction of minimally invasive surgery and enhanced recovery after surgery, there is an increased need for an assessment tool to evaluate the quality of recovery [1]. The quality of recovery is assessed as recovery time, pain, nausea, postoperative complication rates, and adverse events. Although these parameters are essential objective components for the quality of recovery, they do not fully reflect the patient's social and psychological recovery experience. Different patient-centered measurement tools have been developed and validated to evaluate the patient's recovery experience [2-5].
The Quality of Recovery-15 (QoR-15) questionnaire is a short 15-item scale derived from QoR-40 [3]. It assesses five domains of a patient's health: emotional state, physical comfort, psychological support, physical independence, and pain. Each item uses an 11-point numeric rating scale. The sum of the scores of the 15 items ranges from 0 to 150 , with a high score indicating good quality of recovery. The questionnaire decreases the evaluation time, improves convenience, and has strong evidence for good content validity and internal consistency. However, a Korean version of QoR-15 (QoR$15 \mathrm{~K}$ ) has not been established. The development of QoR$15 \mathrm{~K}$ and its validation would improve the quality of recovery after surgery in the Korean population. Therefore, we developed and validated QoR-15K according to the COnsensusbased Standards for the selection of health Measurement INstruments (COSMIN) taxonomy in surgical patients [6]. 


\section{Patients and Methods}

2.1. Translation and Cultural Adaption of QoR-15. After obtaining consent from the original author, two independent translators (native speakers of the Korean language) translated the English version of QoR-15 into Korean. We first made a temporary Korean version of QoR-15, and then, two different translators (native speakers of the English language) back-translated this version into English. Therefore, each question was rendered in its most intelligible form by a committee of four translators. The committee modified "feeling rested" to "feeling rested enough," "doctors and nurses" to "medical personnel," and "feeling comfortable and in control" to "feeling comfortable and going well" in Korean. The committee concluded that these changes made it easier to understand the questionnaire in Korean while conveying the nuances of the English version. After the committee came to a consensus on the Korean version of QoR-15, it was tested on 10 randomly selected postoperative patients. The test revealed that all questions were understandable. As a result, the Korean version of QoR-15 (QoR-15K) was finalized.

2.2. Patients. After the institutional review board approved this research, a prospective observational study was conducted. Patients provided oral consent. Patients with poor Korean comprehension or those with cognitive impairment were excluded from the study. Furthermore, those with a known history of substance abuse, any severe preexisting medical conditions that limited objective assessment after the operation, or any life-threatening postoperative complications were excluded.

Demographic and perioperative data were collected via the hospital's medical information system. We recorded sex, age, American Society of Anesthesiologists physical status score (ASA PS), duration of surgery and anesthesia, extent of surgery, duration of postoperative stay, and postoperative complications within $24 \mathrm{~h}$. We classified the extent of surgery as minor, intermediate, or major. Laparoscopic abdominal surgery with minimal incision lengths and surgery on the surface of the body was classified as minor surgery, laparoscopic abdominal surgery using a hand port as intermediate surgery, and open abdominal surgery as major surgery.

2.3. Protocol. Before the day of surgery, investigators asked the patients to complete the QoR-15K questionnaire as a measure of baseline status. The patients were then asked to repeat the questionnaire at $24 \mathrm{~h}$ postoperatively. Patients were also asked to rate their overall postoperative recovery using an 11-point numerical rating scale (NRS), which ranged from "poor recovery (0)" to "excellent recovery (10)." All patients were asked to repeat the QoR-15K questionnaire within $30-60 \mathrm{~min}$. We selected 50 patients from those who completed the QoR-15K questionnaire. The selected patients were asked to repeat the QoR-15K and SF36 Korean version 2.0 questionnaires on postoperative day 7 to verify the correlation between the two questionnaires.
2.4. Psychometric Evaluation. Domain reliability represents the degree to which the measurement is free from measurement errors. It contains internal consistency, reliability, and measurement error. To assess internal consistency and measurement error, we used Cronbach's $\alpha$ and split-half reliability. To assess reliability, we used test-retest reliability at $24 \mathrm{~h}$ postoperatively. Measurement error was estimated according to COSMIN taxonomy [6].

Domain validity denotes the degree to which an outcome measures the construct of interest. It comprises content, criterion, and construct validities. Content validity is the degree to which an instrument is an adequate reflection of the construct to be measured. It was evaluated via interviews with patients and researchers. To assess criterion validity, we tested predictive validity by calculating the correlation between postoperative hospitalization duration and QoR$15 \mathrm{~K}$ scores at $24 \mathrm{~h}$ postoperatively. Construct validity comprises structural validity, hypothesis testing, and crosscultural validity. Structural validity assesses whether the instrument is an adequate reflection of the dimensionality of the construct to be measured and is determined via exploratory factor analysis. Hypothesis testing examines how much the instrument measure relates to other measures in a way that is expected if the instrument is validly measuring the supposed construct. We hypothesized that the patient who had experienced poor postoperative recovery would have a lower QoR-15K score. We assessed the difference of mean score for QoR-15K according to the patients who had good or poor recovery. An NRS score of recovery $\geq 7$ represented good recovery, while an NRS score of recovery $<7$ represented poor recovery [3]. Cross-cultural validity describes the degree to which a translated or culturally adapted questionnaire is an adequate reflection of the original questionnaire. Because we only translated and changed nuances, we only evaluated the translation process.

Responsiveness is an instrument's ability to measure change over time. Responsiveness was measured via standardized response mean (SRM), which was calculated by dividing the mean change in the score by the standard deviation (SD) of the difference [7]. Cohen's effect size was calculated as the average change in the QoR-15K score (from preoperative to postoperative) divided by the pooled SD [8]. We also performed hypothesis testing to assess responsiveness according to COSMIN taxonomy [6]. We hypothesized a negative correlation between surgery duration and QoR-15K.

Recruitment rate, rate of completion, and time taken to complete QoR-15K were used to test clinical feasibility.

2.5. Assessment of Methodological Quality. The COSMIN four-point checklist, consisting of 10 boxes, was used to assess the methodological quality of the study [9]. Each item in a box is rated as a four-point checklist, and the measurement is determined using the lowest rating of any items in a box. We did not evaluate the patient-reported outcome measure development box because we did not develop QoR-15K.

2.6. Statistical Analysis. We selected a sample size of 150 patients with reference to previous research in the same field 
TABle 1: Demographic data of the patients.

\begin{tabular}{lc}
\hline Characteristics & Value \\
\hline Age (years) & $58.5 \pm 12.8(26-88)$ \\
Sex (male/female) & Male 61.6 \pm 11.7 and female 55.5 \pm 13.0 \\
ASA PS (I/II/III) & $74 / 76$ \\
Type of surgery & $60 / 83 / 7$ \\
$\quad$ Breast surgery & 4 \\
Thyroid surgery & 4 \\
Colorectal surgery & 72 \\
$\quad$ Hepatobiliary surgery & 4 \\
$\quad$ Stomach surgery & 22 \\
Gynecologic surgery & 27 \\
$\quad$ Urologic surgery & 17 \\
Duration of surgery (min) & $169.4 \pm 88.7(25-485)$ \\
Duration of anesthesia (min) & $210.1 \pm 93.9(44-545)$ \\
Length of postoperative hospital stay (days) & $8.4 \pm 6.2(2-49)$ \\
Postoperative complications within 24h of surgery & 21 \\
Preoperative QoR-15K score & $118.3 \pm 24.4(86-150)$ \\
Postoperative QoR-15K score & $84.4 \pm 28.4(18-150)$ \\
\hline
\end{tabular}

ASA PS: American Society of Anesthesiologists performance status; QoR-15K: Korean version of QoR-15. Data are presented as mean \pm standard deviation (range) or frequencies.

and based on the advice of a university statistician [3, 4]. Demographic and perioperative data were presented as means (SD). Qualitative data were presented as frequency and percentage. The Kruskal-Wallis test was used to analyze differences in distribution. Comparisons of the QoR-15K scores were analyzed using the Mann-Whitney $U$ test. Associations were measured using Pearson's correlation coefficient $(r)$ and Spearman's correlation coefficient $(\rho)$. All analyses were performed using SPSS Statistics for Windows, version 21.0 (SPSS; IBM Corp, Armonk, NY). The statistical significance for all analyses was set at $P<0.05$.

\section{Results}

3.1. Patients and Questionnaire Data. Of the 165 patients who were asked to participate in this study, 8 were not eligible, and 2 refused to participate. Five patients were excluded after recruitment due to incomplete questionnaires. Finally, a total of 150 patients completed their pre- and postoperative questionnaires. All patients received general anesthesia. The demographic and clinical characteristics of the 150 patients are given in Table 1.

The completion rate of QoR-15K was $100 \%$ preoperatively and $97 \%$ at $24 \mathrm{~h}$ postoperatively. The mean duration of hospitalization was $8.4 \pm 6.2$ days. It took $157.0 \pm 33.0 \mathrm{~s}$ to complete QoR-15K preoperatively and $138.1 \pm 30.7 \mathrm{~s}$ at $24 \mathrm{~h}$ postoperatively. Most patients were able to complete QoR-15K without assistance. These results show that QoR$15 \mathrm{~K}$ is acceptable and clinically feasible. The baseline and postoperative QoR-15 scores were $118.3 \pm 24.4$ and $84.4 \pm$ 28.4 , respectively, with a significant difference $(P \leq 0.001)$. Postoperative QoR-15K scores were in the range of 18-150. One patient reported a postoperative QoR-15K score of
TABLE 2: Methodological quality ratings based on the COSMIN checklist with four-point scale.

\begin{tabular}{lc}
\hline Box & Rating \\
\hline Content validity & Adequate \\
Structural validity & Adequate \\
Internal consistency & Adequate \\
Cross-cultural validity & Adequate \\
Reliability & Adequate \\
Measurement error & Adequate \\
Criterion validity & Very good \\
Hypothesis testing for construct validity & Very good \\
Responsiveness & Adequate \\
\hline
\end{tabular}

150. There was a positive correlation between age and time taken to complete the questionnaire $(\rho=0.332 ; P \leq$ 0.001 ) and a negative correlation between the total QoR$15 \mathrm{~K}$ score and time taken to complete QoR-15K $(\rho=-0.276 ; P \leq 0.001)$.

3.2. Psychometric Properties. In the domain "Reliability," Cronbach's $\alpha$, standard error of measurement, and splithalf reliability were $0.856,10.78$, and 0.831 , respectively. The test-retest reliability $(\rho)$ was 0.945 (95\% confidence interval [CI]: $0.919-0.962 ; P \leq 0.001)$, and the interclass correlation coefficient test-retest $(\rho)$ was 0.903 (95\% CI: $0.869-$ 0.929; $P \leq 0.001$ ).

In the domain "Validity," content validity, according to the COSMIN four-point checklist, was determined to be adequate (Table 2). We use predictive validity to assess criterion 
validity. The postoperative QoR-15K score negatively correlated with postoperative length of hospital stay $(\rho=0.265$; $P=0.004)$. These results show that a lower QoR-15K score predicts a longer postoperative stay; therefore, QoR-15K has predictive validity. To assess structural validity, we performed exploratory factor analysis, showing acceptable results (Kaiser - Meyer - Olkin measure $=0.821 ; \quad P=0.000)$. To assess construct validity, we performed hypothesis testing as follows. We hypothesized that the patient who had experienced poor postoperative recovery would have a lower QoR$15 \mathrm{~K}$ score. The QoR-15K score significantly differed between the good $(n=82)$ and poor $(n=68)$ recovery groups $(96.2 \pm 25.3$ and $70.2 \pm 25.3(P \leq 0.001)$, respectively). The NRS of recovery showed a positive correlation with postoperative QoR-15K $(\rho=0.579 ; P \leq 0.001)$, demonstrating construct validity. Cross-cultural validity, according to the COSMIN four-point checklist, was determined to be adequate.

In the domain "Responsiveness," we assessed responsiveness and hypothesis testing. Cohen's effect size was 1.28, and SRM was 1.03 (Table 3). These results showed that QoR-15K has a large effect size and high responsiveness. We hypothesized that the patient with longer surgery and anesthesia time would have a lower QoR-15K score. The postoperative QoR$15 \mathrm{~K}$ score negatively correlated with duration of surgery $(\rho=-0.212 ; P=0.009)$ and anesthesia $(\rho=-0.197 ; P=$ $0.016)$. The methodological quality of the study, according to the COSMIN checklist, was rated more than adequate.

We also compared the QoR-15K score with the level of surgery (minor, intermediate, and major). There were significant differences in the distribution of sex, age, ASA physical status score, duration of surgery and anesthesia, and postoperative QoR-15K scores in patients in the minor surgery group (Table 4). The minor surgery group included breast, thyroid, and laparoscopic gynecologic surgeries. Therefore, this group is primarily comprised of women and younger adults. The postoperative QoR-15K scores revealed differences between the three groups $(P=0.004)$. The minor surgery group showed a significantly higher score than the intermediate surgery $(P=0.004)$ and major surgery $(P=0.015)$ groups after post hoc testing using the Tukey HSD test. Twenty-one patients had postoperative complications, including headache, dizziness, fever, and nausea. There were no life-threatening postoperative complications. Postoperative QoR-15K scores between patients with and without postoperative complications did not show a significant difference. According to sex and ASA physical status, there were no significant differences in postoperative QoR-15K scores. In addition, there was also no relationship between the postoperative QoR-15K score and patient's age.

The items within QOR-15 can be divided into two components: physical and mental well-being [3]. The QoR-15K score strongly correlated with the sum of the items in these two components (physical well-being; $\rho=0.913 ; P \leq 0.001$ and mental well-being; $\rho=0.857 ; P \leq 0.001$ ) (Table 5). The QoR-15K score also strongly correlated with the physical component scale $(\rho=0.663 ; P \leq 0.001)$ and moderately with the mental component scale $(\rho=0.44 ; P \leq 0.001)$ of shortform (SF-36) health survey on postoperative day 7 . The sum of physical well-being items on QoR-15K strongly corre- lated with the physical component scale of SF-36 ( $\rho=0.642$; $P \leq 0.001$ ) (Table 6).

\section{Discussion}

In the current study, we assessed QoR-15K in patients recovering from general anesthesia. The results of our study indicate that QoR-15K has acceptable levels of validity, reliability, responsiveness, and clinical feasibility in the Korean population.

Several findings indicate that QoR-15K has acceptable validity. First, the postoperative QoR-15K score showed a negative correlation with the postoperative length of hospital stay. Second, the QoR-15K score showed a significant correlation with recovery NRS. The QoR-15K scores in the good recovery group were significantly higher than those in the poor recovery group. These results are consistent with the original study and support construct validity. Third, the fact that the baseline and postoperative QoR-15K scores were significantly different in this study could be a good indicator of construct validity.

QoR-15K demonstrated excellent internal consistency with Cronbach's $\alpha$ and split-half reliability $[10,11]$ exceeding the recommended value (Cronbach's $\alpha=0.7$ and split - half reliability $=0.75$ ) [12]. These results confirm the acceptable reliability of QoR-15K. Corrected item-total correlation (0.23-0.72) indicates that the items in QoR-15K are appropriate and relevant with no unnecessary items. The corrected item-total correlation of item 13 (0.23), indicating nausea or vomiting, was relatively low in comparison with the original study (0.47). This finding may be related to the relatively low incidence rate of nausea or vomiting in this study. Test-retest reliability $(\rho=0.95)$ was excellent and similar to that in the original study $(\rho=0.99)$. All items in QoR-15K performed at above acceptable levels $(\rho=0.80-0.91)$, which is consistent with the original study [3].

Consistent with previous findings, QoR-15K was not related to demographic factors, such as sex, age, and ASA physical status, in this study $[3,13]$. This result shows that QoR-15 is less affected by these demographic variables. However, QoR-40 differs between sexes, with a higher score for males. This difference indicates that QoR-15 may not reflect the general notion that women typically experience a lower quality of recovery [4].

Responsiveness is also acceptable. The SRM value of the total of the QoR-15K scores was 1.03, demonstrating the ability to assess alterations in the postoperative recovery period [14]. However, the SRM value of the items concerning emotional status (feeling worried or anxious or feeling sad or depressed) assessed in this study was low compared with that in the original research $(0.10$ vs. 0.35 and 0.06 vs. 0.20 , respectively) [3]. A Japanese study also showed lower SRM in emotional status [15]. East Asian people usually express less emotion in response to especially sad situations [16]. We believe this cultural characteristic may have affected our results. QoR-15K had a negative correlation with the length of postoperative hospital stay $(\rho=-0.28)$ and duration of surgery and anesthesia ( $\rho=-0.21$ for both). These associations were similar to the Portuguese version $(\rho=-0.28$ for 


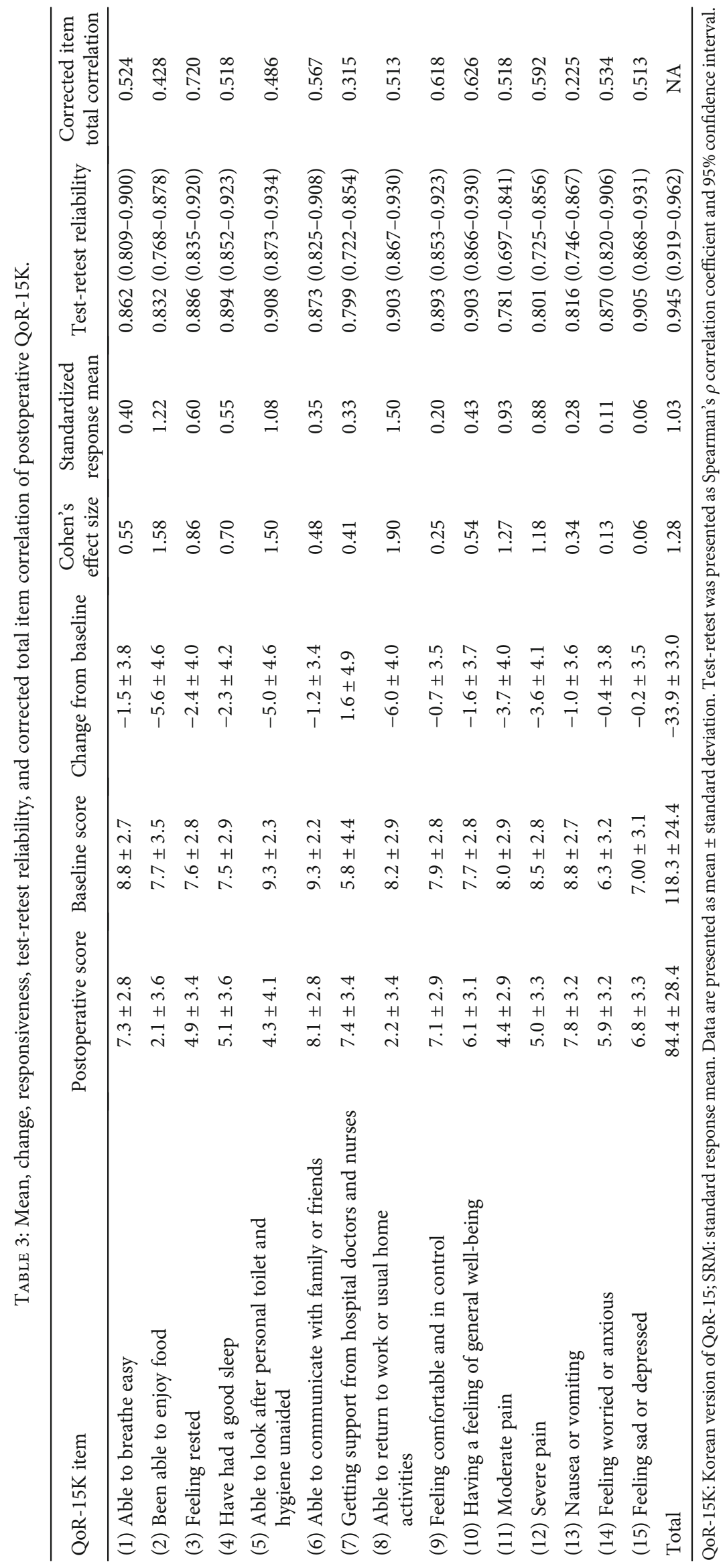


TABLE 4: Patient characteristics according to the extent of surgery.

\begin{tabular}{|c|c|c|c|c|}
\hline Variable & Minor $(n=33)$ & Intermediate $(n=75)$ & Major $(n=42)$ & $P$ value \\
\hline Age & $46.6 \pm 9.9$ & $62.9 \pm 11.4$ & $60.0 \pm 11.2$ & $\leq 0.001$ \\
\hline Sex (male/female) & $2 / 31$ & $45 / 30$ & $27 / 15$ & $\leq 0.001$ \\
\hline ASA PS class (I/II/III) & $21 / 12 / 0$ & $24 / 46 / 5$ & $15 / 25 / 2$ & 0.03 \\
\hline Duration of surgery & $112.2 \pm 61.3$ & $163.3 \pm 85.3$ & $225.2 \pm 81.4$ & $\leq 0.001$ \\
\hline Duration of anesthesia & $144.9 \pm 69.8$ & $208.0 \pm 89.5$ & $264.9 \pm 85.3$ & $\leq 0.001$ \\
\hline Postoperative hospital stay (days) & $5.0 \pm 4.2$ & $8.9 \pm 5.0$ & $10.1 \pm 8.1$ & $\leq 0.001$ \\
\hline Postoperative complications within $24 \mathrm{~h}$ of surgery & 3 & 13 & 5 & 0.28 \\
\hline Preoperative QoR-15K & $124.2 \pm 2$ & $115.4 \pm 25.0$ & $118.6 \pm 26.5$ & 0.22 \\
\hline Postoperative QoR-15K & $98.7 \pm 29.2$ & $80.2 \pm 25.4$ & $80.7 \pm 29.7$ & $\leq 0.001$ \\
\hline
\end{tabular}

ASA PS: American Society of Anesthesiologists performance status; QoR-15K: Korean version of QoR-15. Minor: laparoscopic abdominal surgery with minimal incision lengths and surgery on the surface of the body; intermediate: laparoscopic abdominal surgery using a hand port; major: open abdominal surgery. Data are presented as mean \pm standard deviation (range) or frequencies.

TABLE 5: Interdimensional correlation of QoR-15K.

\begin{tabular}{llll}
\hline & Total QoR-15K & Physical well-being & Mental well-being \\
\hline Physical well-being & $\rho=0.913 ; P \leq 0.001$ & \\
Mental well-being & $\rho=0.857 ; P \leq 0.001$ & $\rho=0.653 ; P \leq 0.001$ & \\
\hline
\end{tabular}

QoR-15K: Korean version of QoR-15. Physical well-being: sum of items of 1, 2, 3, 4, 5, 8, 11, 12, and 13 in QoR-15K; mental well-being: sum of items of 6, 7, 9, 10,14 , and 15 in QoR-15K.

TABLE 6: Correlation between QoR-15K and SF-36 scores.

\begin{tabular}{lcr}
\hline & Shysical component score & Mental component score \\
\hline Total QoR-15K & $\rho=0.663 ; P \leq 0.001$ & $\rho=0.444 ; P=0.001$ \\
Mental well-being & $\rho=0.583 ; P \leq 0.001$ & $\rho=0.443 ; P=0.001$ \\
Physical well-being & $\rho=0.642 ; P \leq 0.001$ & $\rho=0.427 ; P=0.002$ \\
\hline
\end{tabular}

QoR-15K: Korean version of QoR-15; SF-36: short-form health survey Korean version 2.0. Mental well-being: sum of items of 6, 7, 9, 10, 14, and 15 in QoR-15K; physical well-being: sum of items of 1, 2, 3, 4, 5, 8, 11, 12, and 13 in QoR-15K.

the length of hospital stay) yet modestly weaker than those in the original evaluation of QoR-15 ( $\rho=-0.53$ for the length of hospital stay, $\rho=-0.49$ for the duration of surgery).

Most patients were able to complete the QoR-15K questionnaire in $3 \mathrm{~min}$. This result is similar to that of the English and Portuguese versions $[3,13]$. As expected, older participants and patients with lower QoR-15K scores took more time to complete the questionnaire. It is also notable that $97 \%$ of participants completed QoR-15K within $24 \mathrm{~h}$ postoperatively. These data indicate good clinical feasibility.

This study has certain limitations. First, we did not include patients who underwent ambulatory or orthopedic surgeries and a patient who received regional anesthesia. Patients who underwent neurosurgical and cardiac surgeries were also not included in this study. However, our study included patients with all levels of surgery, and we do not believe that these exclusions affected our results. Second, postoperative complications within $24 \mathrm{~h}$ of surgery did not affect the QoR-15K score. We included patients who underwent elective surgery, and there were only a small number of reported complications in this study, which was minor. We believe these results could change if we include patients who undergo emergency surgery and a more significant number of patients.

\section{Conclusion}

In conclusion, we translated QoR-15 into Korean and validated it according to the COSMIN taxonomy. QoR-15K has acceptable validity, reliability, responsiveness, and clinical feasibility and may help in measuring the quality of postoperative recovery in the Korean population undergoing surgery.

\section{Data Availability}

The datasets generated during and/or analyzed during the current study are available from the corresponding author on reasonable request. 


\section{Conflicts of Interest}

The authors declare that there is no conflict of interest regarding the publication of this article.

\section{Authors' Contributions}

All authors made substantial contributions to conception and design, acquisition of data, or analysis and interpretation of data; took part in drafting the article or revising it critically for important intellectual content; agreed to submit to the current journal; gave final approval of the version to be published; and agreed to be accountable for all aspects of the work.

\section{References}

[1] T. E. Miller and M. Mythen, "Successful recovery after major surgery: moving beyond length of stay," Perioperative Medicine, vol. 3, no. 1, pp. 4-6, 2014.

[2] K. B. Kluivers, I. Riphagen, M. E. Vierhout, H. A. M. Brölmann, and H. C. W. de Vet, "Systematic review on recovery specific quality-of-life instruments," Surgery, vol. 143, no. 2, pp. 206-215, 2008.

[3] P. A. Stark, P. S. Myles, and J. A. Burke, "Development and psychometric evaluation of a postoperative quality of recovery score: the QoR-15," Anesthesiology, vol. 118, no. 6, pp. 13321340, 2013.

[4] P. S. Myles, B. Weitkamp, K. Jones, J. Melick, and S. Hensen, "Validity and reliability of a postoperative quality of recovery score: the QoR-40," British Journal of Anaesthesia, vol. 84, no. 1, pp. 11-15, 2000.

[5] B. F. Gornall, P. S. Myles, C. L. Smith et al., "Measurement of quality of recovery using the QoR-40: a quantitative systematic review," British Journal of Anaesthesia, vol. 111, no. 2, pp. 161169, 2013.

[6] L. B. Mokkink, C. B. Terwee, D. L. Patrick et al., "The COSMIN study reached international consensus on taxonomy, terminology, and definitions of measurement properties for health-related patient-reported outcomes," Journal of Clinical Epidemiology, vol. 63, no. 7, pp. 737-745, 2010.

[7] J. A. Husted, R. J. Cook, V. T. Farewell, and D. D. Gladman, "Methods for assessing responsiveness: a critical review and recommendations," Journal of Clinical Epidemiology, vol. 53, no. 5, pp. 459-468, 2000.

[8] J. N. Katz, M. G. Larson, C. B. Phillips, A. H. Fossel, and M. H. Liang, "Comparative measurement sensitivity of short and longer health status instruments," Medical Care, vol. 30, no. 10, pp. 917-925, 1992.

[9] L. B. Mokkink, H. C. W. de Vet, C. A. C. Prinsen et al., "COSMIN risk of bias checklist for systematic reviews of patientreported outcome measures," Quality of Life Research, vol. 27, no. 5, pp. 1171-1179, 2018.

[10] C. B. Terwee, S. D. Bot, M. R. de Boer et al., "Quality criteria were proposed for measurement properties of health status questionnaires," Journal of Clinical Epidemiology, vol. 60, no. 1, pp. 34-42, 2007.

[11] M. Tavakol and R. Dennick, "Making sense of Cronbach's alpha," International Jouranl of Medical Education, vol. 2, pp. 53-55, 2011.
[12] I. McDowell, "The theoretical and technical foundations of heath measurement," in Measuring health: a guide to rating scales and questionnaires, pp. 37-39, Oxford University Press, New York, 3rd edition, 2006.

[13] A. C. Sá, G. Sousa, A. Santos, C. Santos, and F. J. Abelha, "Quality of recovery after anesthesia: validation of the Portuguese version of the 'Quality of Recovery 15' Questionnaire," Acta Médica Portuguesa, vol. 28, no. 5, pp. 567-574, 2015.

[14] R. R. Luiz and R. M. V. Almeida, "On the measurement of change in medical research," International Journal of Statistics in Medical Research, vol. 1, pp. 144-147, 2012.

[15] Y. Tanaka, T. Wakita, S. Fukuhara et al., "Validation of the Japanese version of the quality of recovery score QoR-40," Journal of Anesthesia, vol. 25, no. 4, pp. 509-515, 2011.

[16] Y. E. Chentsova-Dutton, J. P. Chu, J. L. Tsai, J. Rottenberg, J. J. Gross, and I. H. Gotlib, "Depression and emotional reactivity: variation among Asian Americans of East Asian descent and European Americans," Journal of Abnormal Psychology, vol. 116, no. 4, pp. 776-785, 2007. 